ISSN: 1641-4713; e-ISSN: 2081-1160

DOI: https://doi.org/10.36551/2081-1160.2020.25.57-79

\title{
Asociacionismo y cambio social en comunidades rurales andinas. Aproximación al caso de los cafeteros colombianos
}

\author{
Associativism and Social Change in Andean Rural Communities: \\ An Approximation to the Case of Colombian Coffee Growers
}

\author{
Diana María Rodríguez-Herrera \\ Universidad Tecnológica de Pereira, Colombia \\ Becaria Postdoctorial - \\ ID ORCID: https://orcid.org/0000-0003-4156-8586 \\ E-mail: dianarodriguez@utp.edu.co
}

Ministerio de Ciencia, Tecnología e Inovación Colombia e Innogest S.A.S.

Recepción: 16.02 .2020

Aprobación: 13.06.2020

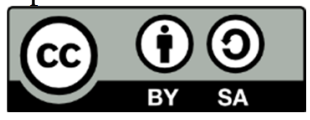

Resumen: El asociacionismo constituye un impulsor de la modernización agraria, crucial en el análisis de procesos de cambio social en comunidades rurales. El presente artículo aporta evidencia etnográfica sobre tipos y prácticas de asociacionismo en comunidades andinas dedicadas al cultivo de café, buscando contribuir a las discusiones relativas a la reformulación del desarrollo en Latinoamérica. El trabajo de campo abordó la cotidianidad de una asociación agrícola el municipio de La Celia (Risaralda, Colombia), con el objetivo de analizar la supuesta debilidad asociativa en comunidades andinas. Los resultados muestran cómo situaciones de crisis económica cafetera tienden a motivar la actualización súbita de formas asociativas locales, al amparo de organismos trasnacionales, que acaban instalando nuevos dilemas productivos al interior de las comunidades. Este fenómeno afecta sociabilidades domésticas, que vertebran el asociacionismo local. Influye también en la confianza social y en la aptitud asociativa futura.

Palabras claves: asociacionismo, cambio social, Andes, ruralidad, caficultura, iniciativas de transformación, transiciones.

Abstract: Associativism is a driver of agrarian modernization, crucial in the analysis of social change processes in rural communities. This article provides ethnographic evidence about types and practices of associativism in communities dedicated to coffee cultivation, in the Colombian Andes. It seeks contribute 
to academic discussions regarding the reformulation of development in Latin America. The field work considered the daily life of an agricultural association in the municipality of La Celia (Risaralda, Colombia), with the objective of analyzing the supposed associative weakness in Andean communities. The results show how situations of coffee economic crisis tend to motivate the sudden updating of local associative forms, under the protection of transnational organizations, who end up installing new productive dilemmas within communities. This phenomenon affects domestic sociabilities, which support local associativism. It also influences social trust and future associative aptitude.

Keywords: associativism, social change, Andes, rurality, coffee growing, transformation initiatives, transitions.

Este artículo es resultado del proyecto de investigación "Iniciativas de Transformación y Asociatividad en comunidades rurales andinas", financiado por el Ministerio de Ciencia, Tecnología e Innovación de Colombia mediante el Programa de Estancias Postdoctorales en entidades del SNCTeI 2019. Entidad receptora: Innogest S.A.S.

\section{INTRODUCCIÓN}

En la zona cafetera colombiana, como en otros contextos andinos, el asociacionismo se concibe institucionalmente como una fórmula deseable para ganar escala productiva, superar condiciones geográficas adversas, aumentar productividad, disminuir riesgos, romper viejos paradigmas y acceder a nuevos mercados. Paradójicamente, la solidez del discurso institucional sobre la pertinencia del asociacionismo andino contrasta con la debilidad de las prácticas asociativas de algunas poblaciones, que tienden a registrar bajas tasas de perdurabilidad de empresas asociativas, baja confianza social y escasa eficiencia en el incremento de la renta de sus socios (Cardona et al., 2015; Gómez Cardona, 2010; Lombo, 2013; Rodríguez-Herrera, 2017; Valenzuela y Causiño, 2000).

Unirse y crear asociaciones agrícolas suele emerger de manera casi espontánea en situaciones de crisis (Gómez Cardona, 2010). Así, bajo la premisa de que el asociacionismo genera sinergias positivas con el mercado y el Estado, a las asociaciones agrícolas -en los lugares investigados- tiende a asignárseles discursivamente capacidad para resolver el problema de la recesión, el desempleo, la descentralización e, incluso, el deterioro ambiental. Esta naturalización de las bondades del asociacionismo, analíticamente, nos lleva a revisar la función social que realmente cumplen estas asociaciones. Y de modo más específico preguntarnos: ¿qué ocurre cuando fracasan?; ¿por qué fracasan?; ¿por qué instituciones y agricultores manifiestan que "a la gente le cuesta asociarse"?; ¿hasta qué 
punto el fracaso del asociacionismo limita el surgimiento de la oposición y crítica política al desarrollo en Latinoamérica?

Desde el análisis sociológico, Valenzuela y Causiño (2000) han argumentado que la debilidad asociativa en sociedades como la chilena-fuertemente atravesada por políticas neoliberales- se compensa con la fortaleza de las relaciones familiares, de manera que se produce una disposición a no confiar en extraños sino solo en el grupo familiar o doméstico. Según estos autores, tal tendencia de sociabilidad doméstica tiende a limitar la capacidad de experimentar vínculos poderosos con personas desconocidas, pudiendo estar influida por la importancia otorgada a la cohabitación familiar, las herencias coloniales, los acelerados procesos de modernización, los altos umbrales de inseguridad o las creencias religiosas, entre otros factores. En términos de Valenzuela y Causiño (2000), la lógica de la reciprocidad - propia del familiarismo ${ }^{1}$ - se sitúa en el foco de las explicaciones sobre las causas de debilidad asociativa. Ello es debido a que condiciona la capacidad de apertura hacia lo que es extraño o a confiar en quien no comparte el mismo origen:

Es la potencia del vínculo familiar la que impide autocomprender la existencia como un suceso individual y solitario que sólo puede trascender hacia la relación con extraños [asociacionismo], vale decir con otros individuos igualmente solitarios. Y es también la intensidad de este vínculo lo que empuja constantemente a privilegiar la lealtad hacia el conocido antes que la confianza hacia el extraño (Valenzuela y Causiño, 2000: 335).

Guardando distancia con este tipo de explicaciones que interpelan la relación entre reciprocidades domésticas y relaciones comunales, Victoria (2019) analiza para el caso colombiano lo que él denomina "tradición asociativa" de los agricultores en el municipio cafetero de Apía, vecino de nuestro contexto de estudio en La Celia. Este autor subraya la fortaleza asociativa fundada en la lógica de la reciprocidad como un rasgo típico de sociedades de frontera que, por lo observado en Apía, han resistido diferentes ciclos modernizadores mediante estrategias "colectivistas" (Victoria, 2019: 81). Aunque este autor niega la denominada tesis igualitaria de la colonización comunal planteada por el historicismo moral; coincide con este historicismo en reconocer la importancia de los vínculos de reciprocidad como una condición histórica clave para autogestionar bienes comunes y lograr la integración de la frontera en la economía de mercado.

\footnotetext{
${ }^{1}$ Para Valenzuela y Causiño (2000) la lógica de la reciprocidad no se encuentra el compromiso personal con la promesa hecha (base de la asociatividad), sino en vínculo establecido a través del acto de dar que obliga a recibir y devolver. En tal sentido, la reciprocidad requiere de la eficiencia vinculante de la presencia y el don -en términos maussianos-; mientras que la asociatividad se basa en la capacidad de creer en lo que no se ve y en confiar una promesa contractualmente asegurada.
} 
Centrando su observación en la organización Asoapía, Victoria (2019) ilustra un comportamiento asociativo esencial, que perdura y resiste en condiciones de desigualdad. Nos habla del asociacionismo cafetero como una respuesta adaptativa, creativa y competitiva de un gran grupo de pequeños agricultores que desde el año 2005 autogestionan la exportación de su propia cosecha de café hacia mercados especializados. A diferencia de la explicación de Valenzuela y Causiño (2000), Victoria (2019) no encuentra en la lógica misma de la reciprocidad una limitación para la confianza social y el asociacionismo. Para Victoria (2019), las principales barreras del procomún son factores de tipo político como el asistencialismo, la corrupción, la violencia política y el corporativismo de la Federación Nacional de Cafeteros de Colombia (FNCC). De ahí que este autor concluya que la permanencia de organizaciones como Asoapía, pese a estas barreras, denota la fortaleza de una asociatividad fundada e impulsada por vínculos domésticos, que no estaría compensando -como sugieren Valenzuela y Causiño (2000)- debilidades asociativas.

Por lo expuesto, puede inferirse que Asoapía constituye para Victoria (2019) una suerte de agricultura cafetera contestada, con bases identitarias, germen de una gran transformación. Sin embargo, considero que la evidencia histórica no es suficiente para llegar a esta conclusión. Se requieren también microanálisis que permitan interrogar las prácticas asociativas, comprendiéndolas en su multiplicidad, dinamismo y grados de ambivalencia. En este sentido, en el presente artículo propongo problematizar -en perspectiva antropológica- tipos y prácticas de asociacionismo entre productores de café.

Analizaré datos provenientes de una investigación realizada entre 2011 y 2016, en el municipio de La Celia. Esta investigación, más extensamente, había comenzado a finales de 2009, con exploraciones en los municipios de Apía, Mistrató, Santuario, Balboa y luego se concentró en el municipio de La Celia. La estadía en terreno en este municipio tuvo una duración total de nueve meses. Las técnicas empleadas fueron la observación participante, la entrevista semi-estructurada y el taller. Se observaron prácticas productivas al interior de ocho explotaciones cafeteras de tipo familiar (menores de 10 hectáreas), seleccionadas mediante un muestreo de caso-tipo, aunque tratadas como muestras de máxima variación. Las fincas se consideraron como sistemas abiertos, por lo que la indagación incluyó seguimiento a algunos espacios de sociabilidad de los que participaban las familias: asociaciones de productores, escuela, Alcaldía, compra-ventas de café, iglesias, fiestas locales. A continuación, los nombres de las personas fueron cambiados por respeto a su privacidad. 


\section{ASOCIACIONISMO EN CONTEXTOS CAFETEROS}

La literatura plantea la condición de frontera como un rasgo característico de la producción cafetera (Topik et al., 2012). Su carácter indefinido, entre plantación forestal y huerto silvestre, generan una estructura de costos que ha facilitado la expansión del café en tierras marginales de montaña bajo regímenes de explotación doméstica, articulados mediante procesos de clusterización (Palacios, 1983; Palacios y Safford, 2002; Topik et al., 2012; Tulet, 2007; Victoria, 2019). Ligado a esta estructura productiva, la orientación exportadora del cultivo de café implica reunir grandes volúmenes del grano, de manera que ningún productor está en condiciones de valerse enteramente por sí mismo, sino que requiere del concurso de los demás. Este espacio de cooperación productiva suele situarse en la intersección entre sociabilidades domésticas y asociacionismo institucionalizado (Gómez Cardona, 2010; Lombo, 2013; London, 1999; Palacios y Safford, 2002; Victoria, 2019).

Para ilustrar cómo ha ido cambiando esta intersección, de manera esquemática identifico dos tipos u olas de asociacionismo en el municipio de La Celia. La primera ola, que surge entre la década de 1920 y la década de 1990, corresponde con la modernización de la industria cafetera y su crisis estructural, cuando aparecen las principales instancias de representación gremial de la Federación Nacional de Cafeteros de Colombia (FNCC): el Comité Departamental de Cafeteros y el Comité Municipal de Cafeteros. También se crea la Cooperativa Municipal de Cafeteros, como derivación de la Cooperativa Departamental de Cafeteros, ambas amparadas por la $\mathrm{FNCC}^{2}$.

Durante esta ola, la FNCC -presentada públicamente como "la ONG rural más grande del mundo" (Ministerio de Cultura y Federación Nacional de Cafeteros de Colombia, 2012: 68)- se convirtió en la principal forma asociativa de los productores de café, que agrupaba a más del $70 \%$ de los agricultores de municipios como La Celia. A través de esta forma de asociacionismo, la FNCC pretendió funcionalizar sociabilidades domésticas propias de la agricultura familiar dentro de la fuerza de trabajo industrial orientado a la exportación, lo cual tendió a fragmentar lazos comunitarios (London, 1999; Palacios y Safford, 2002; Victoria, 2019). Esta incidencia del corporativismo de la FNCC, sin embargo, no ha sido uniforme ni exenta de resistencias. "Estar con y en contra de la FNCC al

\footnotetext{
${ }^{2}$ La FNCC fue creada en 1927 por una élite de empresarios y hacendados antioqueños, con el fin de encontrar una actividad económica sustitutiva a los productos de exportación heredados del periodo colonial, como el tabaco, la quina y el añil (Palacios, 1983; Tulet, 2007).
} 
parecer constituye una estrategia común entre los pequeños productores" (Rodríguez-Herrera, 2017: 197). Ellos, de forma tácita y explícita, sugieren la institucionalización de un sistema creado para apropiarse de su trabajo, el cual paradójicamente se niegan a abandonar formalmente (Rodríguez-Herrera, 2017).

Este tipo de tensiones y resistencias se vienen haciendo más visibles desde la década de 1990, cuando los agricultores quedaron expuestos al libre mercado, conformándose un escenario de crisis, donde situamos la segunda ola del asociacionismo cafetero. Ésta se caracteriza por la emergencia de patrones de "especialización flexible" (Harvey, 1998), ligados al consumo reflexivo, que trajo como consecuencia la diversificación de los procesos de asociatividad. Las nuevas asociaciones agrícolas vendrían a comunicar, de manera tanto o más eficiente que las antiguas asociaciones cafeteras centralizadas en la FNCC, una serie de valores sociales positivos asociados a los denominados "cafés especiales" (Roseberry, 1996); esto es, justicia, equidad, responsabilidad, inclusión, preservación ambiental, cuidado de la salud, etc. Es decir, si el comercio estandarizado de café no tenía una buena imagen, el comercio de especialidad la restauraría (Dicum, 2003; Roseberry, 1996).

\section{CONTEXTO MUNICIPAL DE LA CELIA}

El municipio de La Celia está localizado en la Cordillera Occidental, en límites entre los departamentos de Risaralda, Valle del Cauca y Chocó, a 66 Km de la ciudad de Pereira, que es la capital departamental. Fundada en 1914, La Celia hizo parte del municipio de Santuario hasta $1959^{3}$, cuando fue reconocida como municipio independiente. Entre 1905 y 1966, fue parte del Departamento de Caldas; en 1966 se anexó al límite político-administrativo del Departamento de Risaralda. Los antecedentes de este ordenamiento territorial datan del siglo XIX, cuando el espacio de La Celia hacía parte de la frontera entre el Estado Soberano de Antioquia y el Estado Soberano del Cauca, de acuerdo con la división territorial de la primera independencia de Colombia.

Según Guhl (2008), los colonos de finales del siglo XIX desmontaban tierras cubiertas por bosques y establecían cultivos de subsistencia que iban rotando, hasta que después de varios ciclos de cultivo sembraban los primeros cafétales. Los grandes productores implementaban cultivos de café valiéndose del modelo de aparcería, a través del cual compraban mejoras y se apropiaban

\footnotetext{
${ }^{3}$ El municipio de Santuario hizo parte del municipio de Apía, hacia finales del Siglo XIX.
} 
de cafetales sembrados en parcelas recién desmontadas por familias sin tierra (Guhl, 2008). De acuerdo con fuentes orales, los cultivos de café pudieron establecerse en La Celia entre las décadas de 1920 y 1930 (Rodríguez-Herrera, 2017), correspondiendo con un desarrollo tardío del cultivo, que se introdujo a Colombia en 1840 y que se dispersó en el Departamento de Caldas a partir de 1860 (Palacio, 1983).

En la actualidad, el término municipal La Celia tiene una extensión de 10.700 hectáreas y una población de 8.580 habitantes (DANE, 2005). El 85\% del suelo municipal es agrícola, el $12 \%$ conserva bosques y el $0,6 \%$ presenta otros usos (DNP, 2016). El café ocupa cerca el 51\% del suelo agrícola, los pastos el $43 \%$ y otras actividades tales como el cultivo del plátano, la caña panelera, el aguacate o la mora, y la avicultura, las granjas de cerdos, la ganadería, la apicultura y la piscicultura ocupan el 6\% (Alcaldía de La Celia, 1999; Gobernación de Risaralda, 2018). Los cultivos de café generan el $70 \%$ del empleo municipal, seguido del comercio -4,5\%-, la administración pública -4\%-, el sector de la construcción $1,5 \%-$, el sector transporte $-0,74 \%-$, los hostales y restaurantes $-0,91 \%$ - (Alcaldía de La Celia, 1999).

Según el Comité Departamental de Cafeteros de La Celia (2016), en el municipio existen alrededor de 1.373 productores de café, con extensiones entre 0,07 y 270 hectáreas. El 94\% de los productores tiene menos de 16 hectáreas y ocupa $66 \%$ del área total; el $81 \%$ tiene menos de 8 hectáreas y ocupa el 30\% del área total; mientras que el 56\% tiene menos de 3,8 hectáreas y ocupa el 10\% del área total. Según un diagnóstico realizado por la Alcaldía de La Celia (2011), los principales problemas identificados por los habitantes rurales son el mal estado de viviendas, el deterioro de las vías, la baja cobertura de la Seguridad Social, los bajos precios del café, el incremento de plagas, el alto costo de fertilizantes, los factores climáticos y la deforestación.

La Celia presenta un índice de pobreza multidimensional del 68\%, por encima del índice nacional que es del $27 \%$. Este indicador de pobreza está ligado a otros indicadores específicos como la tasa de empleo informal -94\%-, la tasa de dependencia económica -36\%-, la tasa de empleo infantil -11\%-, el déficit cualitativo de vivienda $-45 \%$-, la cobertura neta de educación media -33\%- o la tasa de analfabetismo -12\%-(CONPES, 2014; DNP, 2014, 2016). Cabe resaltar que estas tasas de empleo y educación concuerdan con el análisis de Rocha (2014), quien indica que la informalidad laboral caracteriza al 82\% de los ocupados cafeteros en Colombia. Para este autor, en el escenario actual de bajos precios internacionales, las poblaciones productoras especializadas en café conforman un 
mercado laboral de bajos salarios, intensivo en mano de obra no calificada que tiende a integrarse con el mercado laboral urbano (Rocha, 2014).

Como soluciones anticrisis, desde la década del 2000 la FNCC y la Cooperativa Municipal de Caficultores de La Celia impulsaron entre los pequeños cultivadores de café procesos de certificación agrícola (Rodríguez-Herrera, 2017). En 2006, 42 fincas estaban certificadas con el sello Fairtrade y 32 fincas estaban en proceso de certificación con distintos sellos: Fairtrade, Rain Forest Alliance y Nevado. Diez años después, en 2016, este proceso completaría 795 fincas certificadas $-66 \%$ del total municipal- bajo diferentes sellos (RodríguezHerrera, 2017) (Tabla 1).

TABla 1. Producción de CAFÉS ESPECIALES EN LA CELIA, 2006-2016.

\begin{tabular}{|l|c|c|c|c|}
\hline \multicolumn{2}{|c|}{} & \multicolumn{2}{c|}{2006} & $\mathbf{2 0 1 6}$ \\
\hline \multirow{2}{*}{ Agente promotor } & Sellos & $\begin{array}{c}\text { No } \\
\text { de fincas } \\
\text { certificadas }\end{array}$ & $\begin{array}{c}\text { No de fincas } \\
\text { en proceso }\end{array}$ & $\begin{array}{c}\text { No } \\
\text { de fincas } \\
\text { certificadas }\end{array}$ \\
\hline \multirow{2}{*}{$\begin{array}{l}\text { FNCC y } \\
\text { Cooperativa Municipal } \\
\text { de Cafeteros de La Ce- } \\
\text { lia }\end{array}$} & Fairtrade & 42 & 18 & 330 \\
\cline { 2 - 5 } & Rain Forest & 0 & 6 & \\
\cline { 2 - 5 } & Nevado & 0 & 8 & \\
\cline { 2 - 5 } & $\begin{array}{c}\text { Código } \\
\text { de Conducta 4C }\end{array}$ & 0 & 0 & 461 \\
\hline $\begin{array}{l}\text { Agrosolidaria y Funda- } \\
\text { ción Hanns R. Neu- } \\
\text { mann Stiftung }\end{array}$ & ETZ & 0 & 0 & 420 \\
\hline $\begin{array}{l}\text { Servicio Nacional de } \\
\text { Aprendizaje y Coopera- } \\
\text { tiva Municipal de Cafi- } \\
\text { cultores de La Celia }\end{array}$ & Ninguno & 0 & 0 & 42 \\
\hline AMCECAFE & Ninguno & 0 & 0 & 32 \\
\hline Total & & $\mathbf{4 2}$ & $\mathbf{3 2}$ & $\mathbf{9 4 7}$ \\
\hline
\end{tabular}

Fuente: Comité Municipal de Cafeteros de La Celia (2016) y fuentes orales.

Paralelamente, en 2010, fue creada una seccional de la Asociación de Prosumidores Agroecológicos -Agrosolidaria-, de orientación campesina y proambiental $^{4}$, sumándose al mercado de los cafés especiales promovido en La Celia.

\footnotetext{
${ }^{4}$ Esta asociación proviene de la Confederación de Prosumidores Agroecológico -Agrosolidaria-, fundada en 1994 en el departamento de Boyacá, que en la actualidad asocia alrededor de 26.000 agricultores en todo el país (Celeita, 2012).
} 
En 2016, Agrosolidaria tenía 119 productores de café asociados, con procesos de certificación en la marca Fairtrade. Ese mismo año, al amparo del Servicio Nacional de Aprendizaje y de Cooperativa Municipal de Caficultores de La Celia, surgió la iniciativa de otra asociación de pequeños productores en búsqueda de nuevos mercados: la Asociación de Mujeres Cafeteras de La Celia (AMCECAFÉ), de orientación feminista. En 2019, AMCECAFÉ tenía 32 socias, estaba legalmente formalizada, comercializaba café con una marca propia, aunque ninguna de las socias adelantaban aún procesos de certificación agrícola (Tabla 1).

\section{DILEMAS, PRÁCTICAS Y CAPTURAS DEL ASOCIACIONISMO AGRÍCOLA}

La desregularización del mercado ha evidenciado qué tan atrapados están los agricultores colombianos en el monocultivo de café. Después de tres décadas de libre mercado, los elevados costos de reemplazo de las plantaciones refuerzan la dependencia de una tecnología y de un modelo institucional instalado, reduciendo las posibilidades individuales y sociales de cualquier estrategia disruptiva. Tras probar y acumular fracasos con otros cultivos -tales como el plátano, las hortalizas o las frutas- y con la ganadería, los pequeños productores de La Celia continúan sembrando café porque no encuentran otra opción. El café es el cultivo mejor conocido, el único con infraestructura para su procesamiento, líneas de crédito, canales de comercialización y un tratamiento especial dentro de la política agraria nacional. En pocas palabras, constituye un régimen productivo.

Esta forma de enclaustramiento productivo no significa parálisis sino, por el contrario, un intenso activismo en las comunidades que buscan adaptarse. En un trabajo anterior (Rodríguez-Herrera, 2017), hemos demostrado como la pluriactividad, la economía de los cuidados y las tácticas cotidianas constituyen la contracara de la estrategia cafetera, debido a que dotan de elasticidad y dinamismo a este modelo productivo. También ilustramos que si bien, desde un punto de vista social, se juzga como locuras los experimentos agroecológicos de los pequeños agricultores, los experimentos agroindustriales de los grandes productores cafeteros suelen significarse como innovaciones modélicas, de modo que ciertas maneras de reinventar el presente tendrán mas garantías que otras (Rodríguez-Herrera, 2017). En el presente artículo revisamos estos materiales de campo, buscando analizar en qué momento campesinos históricamente estigmatizados se convierten en interlocutores válidos para el Estado y el mercado, qué tipo de propuestas adquieren mayor legitimidad y en qué medida las iniciativas asociativas campesinas tienden a internalizar los efectos negativos de la estrategia cafetera. 
Focalizamos en el caso de Agrosolidaria, una asociación que nació y creció mientras realizaba mi trabajo de campo en el municipio de La Celia, entre 2011 y 2016. En su momento, la creación de Agrosolidaria en La Celia pretendió responder integralmente a una atmosfera política y socioeconómica de dependencia del cultivo de café, caracterizada por cafetales envejecidos, inseguridad alimentaria, afectaciones climáticas ${ }^{5}$, problemas fitosanitarios, ausencia de subsidios agrícolas, endeudamiento generalizado, expectativa de mejorar ingresos agrícolas, desconfianza ante nuevas propuestas productivas, deslegitimación de la FNCC y debilidad asociativa.

La respuesta estatal a esta problemática fueron los programas masivos de renovación de cafetales (Silva, 2012), que significaron a nivel local una profundización del consumo de crédito bancario, introducción de nuevas variedades ciclo más corto, uso intensivo de agroquímicos, que a mediano plazo se reflejaría en incremento de la producción (Rodríguez-Herrera, 2017). Para los precursores de Agrosolidaría esta forma de desarrollo no solucionaba la crisis económica, social y ambiental de municipios cafeteros como La Celia. En su consideración, profundizar la especialización productiva no era la mejor forma de aprovechar los potenciales territoriales y superar la precariedad de la agricultura familiar basada en el café.

Uno de los argumentos que impulsaron la creación de Agrosolidaria sostenía que la mayoría de alimentos ofertados en mercado municipal provenían de lugares lejanos, aunque podían cultivarse en la zona. Se ilustraba el caso de alimentos viajeros, como el plátano, producidos a nivel local pero que eran comercializados en la central de abastos de Pereira, capital departamental, para luego volver al municipio de origen. Como alternativa, Agrosolidaria proponía un proceso autogestionado de circuitos económicos solidarios, basados en la práctica de la agroecología, la economía solidaria, la articulación entre asociaciones agrícolas locales, así como entre productores y consumidores. En estos términos explicó Rafael, maestro y exalcalde municipal de Apía, el origen de Agrosolidaria en Risaralda:

Al finalizar la alcaldía [periodo de gobierno 2004-2008] vimos que no había forma de fortalecer organizaciones dispersas sino que había que agremiarlas. Tuvimos un apoyo de DANSOCIAL (Superintendencia de Economía Solidaria). Ellos nos conectaron con la

\footnotetext{
${ }^{5}$ Mediante el Decreto 020 de 2011, el gobierno de Colombia se declaró en estado de emergencia económica, social y ecológica, debido a "los desastres naturales de dimensiones extraordinarias causados por el fenómeno de la Niña generados de manera posterior a la expedición del Decreto 4580 de 7 de diciembre de 2010 que también declaró el Estado de Emergencia [...] por el termino de 30 días".
} 
Confederación Agrosolidaria de Boyacá, que llevaban 14 años desarrollando una propuesta para el sector solidario rural (Diario de campo, AP_26.12.09).

Definida como una confederación de prosumidores agroecológicos, Agrosolidaria nació en el municipio de Tibasosa, departamento de Boyacá, en 1994, siendo un programa de la Asociación para el Desarrollo Sostenible "Semillas" para integrar familias agricultoras, procesadoras y distribuidoras de productos agrícolas. Agrosolidaria apareció en Risaralda como un mecanismo para dar respuesta a las iniciativas de cambio de personas como Rafael, qua a largo plazo pretendían una transformación inspirada en los movimientos de monedas complementarias en comunidades rurales de Venezuela y Ecuador. Rafael había visitado la Fundación Comercializando como Hermanos de Barquisimeto, en la vecina Venezuela, que tenía una trayectoria de 40 años y "ya tenía supermercados", por lo cual la consideraba un modelo a seguir para crear una red nacional de economía solidaria en Colombia (Diario de campo, AP_26.12.09).

En 2008, Rafael había dinamizado en Apía la creación de la primera seccional de Agrosolidaria en Risaralda. En 2009, estaba promoviendo una ruta solidaria en los municipios de Mistrató, Belén de Umbría y La Celia. No obstante, él reconocía que en Apía era muy difícil que este tipo de iniciativas "echaran raíces" porque el problema "es cultural" y requería una transformación "profunda". Tal dificultad se hizo visible en nuestras primeras aproximaciones a la sede de esta asociación en Apía, que nos sugirieron un bajo consumo de productos justos, en los términos planteados por Agrosolidaria. La sede tenía una pequeña oficina y un mostrador con unos pocos productos ecológicos -miel, panela, mantequilla de maní, vino de naranja, café, chocolates, artesanías, naranjas-, al cual solo llegó un comprador preguntando por mielm durante cerca de dos horas que estuvimos reunidos hablando (Diario de campo, AP_29.01.10).

La baja incidencia del consumo consciente en estos municipios volvió a aparecer durante nuestras observaciones en el municipio de La Celia. Allí, en 2012, los socios de Agrosolidaria habían instalado en el parque principal una carpa verde y amarilla, con los logos de Agrosolidaria y el lema: "Hasta que tengamos una Colombia justa, debemos tener una solidaria" (Diario de campo, CU_19.02.12). Cada domingo vendían diferentes productos, buscando mejorar los ingresos de los socios: calabaza, mora, yuca, lulo, tomate, miel, uniformes para colegio, artesanías. Como parte de la organización de este denominado Ecomercado los asociados asistían a reuniones, recibían capacitaciones, también fabricaban artesanías y cosméticos. Pese a los esfuerzos, con el trascurrir de los meses, dicho mercado ecológico dejó de crecer. Para algunos socios, las bajas 
ventas no "compensaban" el esfuerzo de transportar productos perecederos desde sus fincas. En resumen, su propuesta de diversificación productiva y autoabastecimiento mediante el Ecomercado parecían no prosperar.

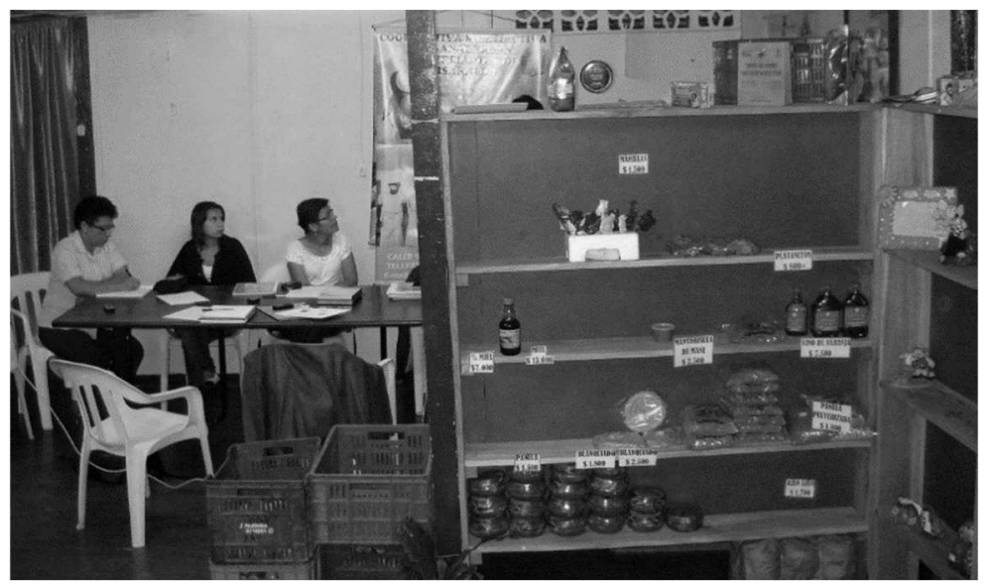

Foto 1. Sede DE AgRosolidaria SECCIONAL APÍA. FUENTE: PROPIA.

Dada la dificultad para establecer un mercado ecológico local, los socios de Agrosolidaria La Celia encontraron en la comercialización de cafés especiales una opción inmediata para mejorar sus ingresos. Es así como pocos meses después de creada esta organización (2010) establecieron transacciones -enunciadas como ayudas - con agentes trasnacionales especializados. La primera de ellas fue la Fundación Hanns R. Neumann Stiftung ${ }^{6}$, que operaba en el marco de un programa de cooperación financiado con dineros de KfW Bankengruppe, conocido en la zona como el Banco Alemán. A través de esta Fundación los socios de Agrosolidaria comenzaron a vender su café a la Asociación de Café Tim Hortons, que a su vez abastecía a la cadena canadiense de cafeterías Tim Hortons.

\footnotetext{
${ }^{6}$ Esta es una fundación impulsada por el Neumann Kaffee Gruppe-NKG-, el cual es un consorcio dedicado a la comercialización de café con sede en la ciudad alemana de Hamburgo.
} 


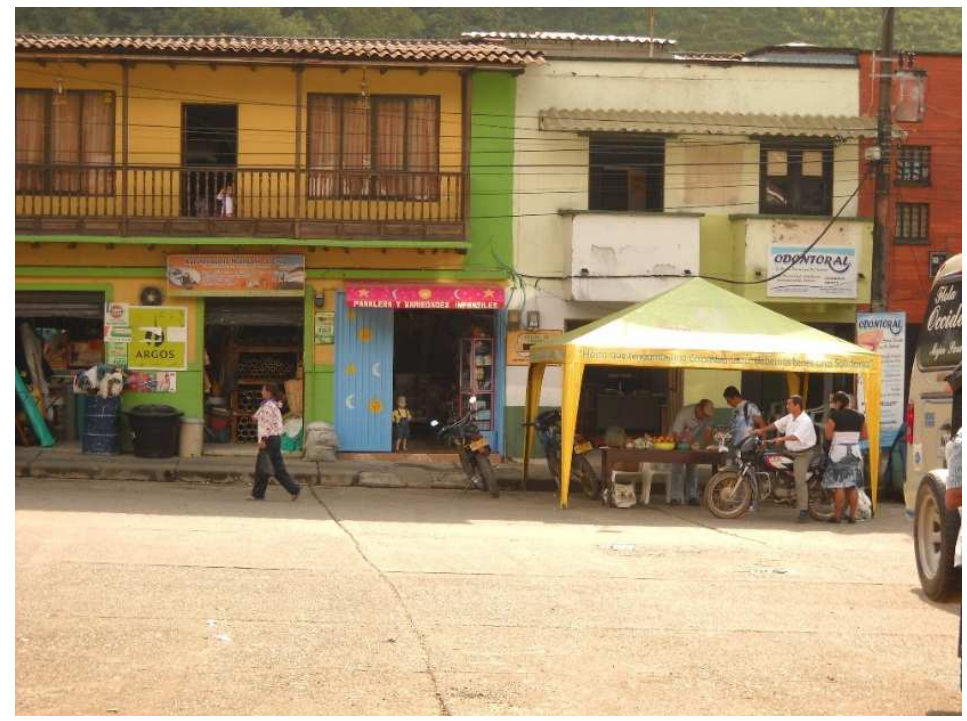

Foto 2. ECOMERCADo EN El MUNiCiPio de LA CELIA, 2012. FuENTE: PROPIA.

Estos negocios derivaron en un proceso de estandarización de procedimientos administrativos, contables y comerciales de la asociación, orientados a la distribución de café. La asociación también emprendió un proceso de reforma de las prácticas productivas de los socios buscando obtener un estándar conocido como taza limpia, el cual seguía criterios gustativos sin comprometerse con estándares ambientales. Es decir, limpio no significaba ecológico. Por lo tanto, atrás parecía quedar el sueño de lograr autosuficiencia local a través de la agroecología o, por lo menos, se postergaba, resignificándose en términos del mercado ético cafetero, considerado como parte del tránsito hacia la práctica agroecológica. Así lo ilustra una entrevista a un directivo de esta asociación realizada en 2016:

Yo me pregunto ¿qué es Agroecología? Como yo soy ignorante, yo no sé nada de Agroecología. Lo poco que yo he entendido es que es una cultura, que se vuelve un estilo de vida, que si es Agroecología entonces debe ser aceptado por la gente. Tendrá entonces que mirar entre eso cómo puede ser viable. Que en últimas si es viable, entonces cómo puede ser rentable. Si eso es Agroecología, yo tengo que decirle a usted: es una cuestión de cultura. Y usted sabe ¿cuál es la cultura del bendito municipio de La Celia?: cafetero. Y lo malo es que es cafetero químico. Entonces usted sabe cuánto tiempo va a tardar para que nosotros como Agrosolidaria - una asociación de prosumidores, o sea, de consumo y productores agroecológicos- me puede decir ¿cuánto vamos a tardar? Entonces qué me pueden reclamar a escasos cinco años [de fundada]» (Entrevista, Mauricio, 47 años, asociado de Agrosolidaria, CU_27.01.16).

El relativo éxito comercial de Agrosolidaria -que en 2016 comercializaba cerca del $5 \%$ de la cosecha municipal de café- no dejaba de despertar críticas 
entre algunos socios, para quienes la especialización en cafés especiales equivalía a "perder el norte"; pues borraba de la propuesta los aspectos "sociales" y de "diversificación" productiva, al tiempo que marcaba la cooptación de la iniciativa por parte de la Fundación Newman. Como señaló una entrevistada: "los alemanes entienden la parte social de manera muy diferente a como la entendemos nosotros" (Diario de campo, LM_02.03.13).

Mas allá del riesgo de banalizar los principios agroecológicos, los directivos de la asociación estaban ilusionados con la posibilidad de superar el estancamiento, expandiendo su agricultura hacia nuevos mercados. Fue así como las primeras ventas de café estabilizaron las expectativas de la mayoría de los asociados, quienes manifestaron que se trataba de retos "nuevos" y "difíciles", para los cuales se estaban "capacitando" gracias a la "ayuda" de la Fundación Newman (Diario de campo, LM_02.03.13). Con el tiempo expresaron tener muchas ocupaciones administrativas, capacitaciones y delegaciones en Agrosolidaria, que los estaban llevando a "descuidar" su propia finca de café (Diario de campo, CU_27.01.16).

Estas vicisitudes en el interior de Agrosolidaria contrastaban con un reconocimiento externo que añadía valores positivos a la producción, mercadeables entre consumidores aparentemente interesados en compensar injusticias sociales con un pequeño sobrecosto en su compra. ${ }^{7}$ Por otro lado, a nivel local, varios productores de café, asociados y no asociados, celebraban el surgimiento de Agrosolidaria porque ofrecía una alternativa comercial a los clásicos canales de la FNCC y los agentes privados en La Celia.

Además de diversificar canales, esta asociación planteaba un trato horizontal entre pequeños agricultores que estaban aprendiendo a mercadear café en un marco de cooperación. Este modo de relación "entre iguales" contrastaba con el carácter unidireccional, jerárquico y especulativo que solían dominar el vínculo entre productores y comercializadores. Así lo resume una entrevistada, Mercedes, juzgando un fallo en el cálculo del precio de su cosecha al momento de venderla a Agrosolidaria: "No es intencional [...] Como ellos son así, poquitos, son gente de campo también, están aprendiendo" (Entrevista, Mercedes, 50 años, agricultora, V9_12.02.16).

\footnotetext{
${ }^{7}$ Así puede verse reflejado en la imagen de la cadena de cafeterías Tim Hortons, basada en la filosofía de marcar una "verdadera diferencia" en materia de sostenibilidad (Tim Hortons, 2014), proporcionando "las habilidades y herramientas que los agricultores necesitan para sobrevivir en tiempos de mercados pobres y prosperar en los buenos" (http://sustainabilityreport.timhortons.com/, traducción propia).
} 
La interpretación que hace Mercedes al momento de la entrevista pareciera tener un fundamento comunal, que le permite situar la identidad de grupo por encima del interés particular. Al parecer, asociarse ponía a prueba la disposición de los agricultores de La Celia a confiar en los otros conocidos desde siempre, tales como familiares, amigos y vecinos. No obstante, la verdadera necesidad de confiar surgía ante la promesa de agentes de mercado extraños y distantes, que a la vez desconfiaban de la agricultura y la moralidad de los socios de Agrosolidaria.

En consecuencia, aparecían en escena intermediarios como la Fundación Hanns R. Neumann Stiftung y la Asociación de Café Tim Hortons, para entregar "ayudas" y "acompañamientos" tecno-administrativos a los agricultores; al tiempo que auditaban la calidad y ética del trabajo productivo y organizativo de la asociación, lo cual implicaba minuciosos formalismos -reuniones, capacitaciones, cataciones de café, elaboración de registros, modificación de infraestructuras, certificaciones agrícolas, etc.-, que -como hemos dicho- permitían funcionalizar reciprocidades locales y, a la vez, transmitir confianza a consumidores lejanos, ansiosos de experimentar la sensación de estar solucionando problemas sociales a través de su compra. Estos formalismos representaron una carga laboral creciente para los agricultores, quienes después de varios ciclos de mercado acabaron sospechando que el sobreprecio ofrecido era muy bajo en relación con las nuevas exigencias, de manera que posiblemente en la asociación estaban trabajando "gratis" (Diario de campo, V1_21.04.16).

Quedaba así bajo sospecha la sinergia entre asociacionismo solidario y mercado cafetero, dejando de ser un espacio confiable y esperanzador, para convertirse en uno cargado de amenazas, recelos y trampas. Una especie de burbuja que podría estallar en algún momento. Como efectivamente ocurrió en 2016, cuando surgieron rumores de nepotismo y malversación de fondos por parte de algunos directivos de la asociación. Estos rumores llevaron a la Fundación Newman a amenazar con suspender o mermar sus procesos de "acompañamiento" empresarial, dejando indirectamente instaladas disputas entre familiares, vecinos y amigos, tal como sucedió durante una asamblea general de Agrosolidaria (Diario de campo, CU_02.04.16).

Asistíamos así a la renovación de la clásica fórmula del asistencialismo cafetero, basada en el productivismo, el paternalismo y la funcionalización de relaciones domésticas propias de la agricultura familiar (Cruz-Rodríguez, 2013; London, 1999; Rodríguez-Herrera, 2017). Pero esta vez orquestada no por el Estado, sino por redes trasnacionales de formación de valor que buscaban simular un mercado ético auténtico, a costa de sobrecargas laborales invisibles, instrumentalización de reciprocidades, tiempo expropiado e invasiones a la vida privada de grupos familiares rurales. 


\section{DE LA GRAN TRANSFORMACIÓN A LA PEQUEÑA REFORMA}

Creadas para enfrentar situaciones de crisis económica y de ausencia del Estado, asociaciones de pequeños agricultores andinos, como la analizada, parecen establecer una interlocución válida con el mercado solo cuando logran adaptar sus iniciativas en términos del comercio mundial. Lo cual tiende a generar extraños ejercicios interpretativos que pueden terminar socavando el contenido central de iniciativas asociativas locales. En el caso de Agrosolidaria, ello implicó una flexibilización y simplificación de ideales de cambio relacionados con la diversificación productiva, la producción ecológica y el autoabastecimiento.

Para algunos habitantes locales esta modificación supuso desdibujar la iniciativa planteada; para otros, una reforma realista de prioridades en función de su aceptabilidad social y viabilidad económica. En nuestra consideración, inicialmente se trató de una iniciativa radical, robusta, de base ambiental, que privilegiaba el futuro en relación con el presente. En consecuencia, demandaba un riguroso escrutinio en las estrategias, las reglas del juego y la moralidad de la propuesta; al mismo tiempo que soportaba gran presión social por mostrar transformaciones concretas de forma inmediata. Frente a esta disyuntiva entre ideales importantes y demandas urgentes, la asociación terminó estabilizando las expectativas productivistas de los socios mediante la participación en el mercado ético del café. Una participación que implicó poner el corazón de la iniciativa entre paréntesis.

Puede entenderse hasta aquí que esta organización siguió una serie de pasos legítimos en términos asociativos, que terminaron restándole alcance y, por lo tanto, legitimidad a su propuesta ambiental. Tras incursionar en el mercado ético del café, la discusión se centró en el tipo de agroecología más razonable para el contexto de La Celia, así como en la duración de la transición agroecológica. Los estándares ambientales inicialmente planteados se alivianaron, en términos de tolerancia a diferencias culturales de los agricultores de La Celia, representados discursivamente como, inherentemente, cafeteros químicos. Una distinción esencial que disponía más para la reproducción que para el cambio, abriendo la puerta a simulacros agenciados por trasnacionales de café, especializadas en diseñar experiencias para el mercado de la moral.

Sin pretender leer el devenir de Agrosolidaria La Celia como una historia cerrada, regido por la lógica del todo o nada, cabe resaltar algunos indicios de transición agroecológica y solidaria que siguen presentes en su discurso y práctica; tales como la existencia del Ecomercado, de un fondo rotativo de ahorro, un proyecto de comercialización de plátano, un proyecto de producción de plantas 
medicinales y aromáticas, así como un proyecto de suministro de alimentos a restaurantes escolares locales. Este tipo de acciones cobran un peso político importante en relación con el núcleo de la iniciativa de Agrosolidaria. No obstante, según nuestras observaciones, se trata de acciones fluctuantes y de pequeña escala, que en la actualidad no alcanzan el mismo peso económico, ni político, de la estrategia cafetera.

Lo registrado en La Celia concuerda, en este sentido, con etnografías como la de Gómez Cardona (2010) y Lombo (2013), que evidencian la desestructuración de la acción colectiva, así como apropiación de luchas y demandas históricas de movimientos sociales de agricultores y mujeres por parte de Estados nacionales, organizaciones trasnacionales y empresas privadas relacionadas con la industria cafetera. En particular, coincidimos con Gómez Cardona (2010), quien señala que iniciativas como las de "cafés orgánicos" se ven catalizadas durante periodos de bajos precios constituidos como "crisis", donde las asociaciones locales de productores deben afrontar de manera rápida y no gradual.

Discutimos, sin embargo, la interpretación de Alzate (2019: 41), para quien Agrosolidaria La Celia ha logrado importantes avances "culturales y políticos" en el comercio justo, con proyecciones en la gobernanza territorial. Este autor describe a Agrosolidaria como una "innovación social” aún incipiente, no asistencialista, que cabe valorar como movimiento organizado de apropiación territorial y contestación productiva que ofrece respuestas concretas a la difícil situación del campo colombiano. De acuerdo con Alzate (2019), la lucha política de esta asociación se refleja en una serie de pequeñas acciones que hacen parte un proceso mayor de transformación del perfil productivo municipal. Si bien sus conclusiones son optimistas y esperanzadoras, posiblemente se quedan en un nivel discursivo, apegado al modo como oficialmente se auto-representa y mercadea esta asociación. En estos términos se expresa:

Con asistencia técnica, un comité de trabajo para la mejora y certificación del sitio, un Fondo Rotatorio y un punto de venta fijo, se ha avanzado en el bienestar y la cohesión social, bajo principios de solidaridad. También, a través del fortalecimiento específico de productos con plátano, que actualmente $\mathrm{y}$, a partir de un proceso de colectivización, regula el precio de mercado y con aumentos notables en la rentabilidad (en más del 70-80\%) y el volumen (480 toneladas / mes) y, café, con mejoras en la calidad, mayores garantías en la producción y venta de este producto. Además de participar en el mercado institucional de alimentos en restaurantes escolares, para el cual se compraron huertas e insumos ( $\mathrm{Al}-$ zate, 2019: 41, traducción propia).

Con todo, Alzate (2019) indica "fragilidad" en la estructura administrativa de esta asociación, sugiriendo que no están operando completamente ciertos procesos técnicos, organizacionales, de "vigilancia y control real" (Alzate, 2019: 52, 
traducción propia). Esta conclusión guarda relación con nuestros registros, así como con el análisis de Hernández, Cardona y Nova (2015), para quienes las asociaciones agrícolas del Departamento de Risaralda -incluyendo Agrosolidaria La Celia- presentan "debilidad de sus modelos gerenciales y en la baja capacidad de gestión gerencial, organizacional y en la cultura de individualismo [sic] que aún prevalece en el sector agropecuario" (Cardona et al., 2015: 12).

Podría pensarse, con Cardona et al. (2015) y Alzate (2019), que bastaría fortalecer las capacidades administrativas y tecnológicas de estas asociaciones para hacerlas competitivas en los mercados nacionales e internacionales. Más aún cuando, según Cardona et al. (2015: 6):

Los líderes de grupos asociativos, concluyen que para enfrentar los tratados de libre comercio, la globalización y la internacionalización de la economía, se necesita un trabajo riguroso de formación aplicada de teorías organizacionales y administrativas, con enfoque asociativo.

No obstante, nuestro análisis nos lleva a concluir que fortalecer ese tipo de capacidades a nivel operativo, instaurando las denominadas "culturas de la auditoría" (Strathern, 2000), no tiende a reflejarse en cambios sociales sustanciales. Por el contrario, puede terminar erosionando la confianza social y las propias iniciativas de transformación, así se alcancen metas locales relacionadas con acceder a fondos o a mercados internacionales. No se trata de un asunto meramente operativo o de conocimiento, sino de uno fundamentalmente estratégico, político y moral, relacionado con posibilitar el despliegue de "diseños autónomos" (Escobar, 2016a), que contrarresten procesos internos de concentración del poder, captura por parte de agentes externos y ausencia de mecanismos para hacer cumplir las reglas.

\section{CONCLUSIÓN}

¿Cómo pensar las reformulaciones del desarrollo en Latinoamérica? Nuestro acercamiento a comunidades andinas ancladas en la industria cafetera implica considerar formas económicas locales siempre en hibridación con el mercado mundial. Permeadas dichas comunidades por un desarrollismo invasivo, están situadas, además, en un país con una dramática realidad humanitaria. Estamos hablando entonces de un contexto geopolítico de frontera (Victoria, 2019), donde resulta estéril la oposición binaria entre "desarrollo alternativo" y "alternativas al desarrollo" (Escobar, 2012). 
En primer lugar, porque no permite captar como ambas se entrecruzan en la vida cotidiana. En segundo lugar, porque implica negar el desarrollo y, como dice Escobar (2016b), el desarrollismo "está en todas partes" de manera que no podemos situarnos "fuera" de él. En tercer lugar, porque el binarismo sugiere la existencia de proyectos de desarrollo acabados, cuando lo común es encontrar propuestas incipientes, contradictorias, inestables, improvisadas, oportunistas, más que planes consistentes de actuación, como bien ilustra el caso de Agrosolidaria La Celia.

Buscando esquivar esta oposición infructuosa, hemos tomado prestado el concepto de iniciativas de transformación, empleado por Hummel (2019, con base en Escobar, 2012) para describir estrategias y narrativas de cambio construidas de abajo hacia arriba. Este concepto evita imponer categorías de desarrollo y permite considerar propuestas de cambio, reconociendo su carácter procesual. No obstante, presupone iniciativas que nacen desde abajo hacia arriba, lo cual no ocurre de manera compacta, sino que puede seguir trayectorias polivalentes e indeterminadas.

En el caso analizado, la iniciativa nació desde arriba, ya que fue sugerida por la Superintendencia de Economía Solidaria y un alcalde local, pero finalmente articuló la acción colectiva local con una organización de base nacional, la Confederación Agrosolidaria, conectándose asimismo con movimientos sociales de otros países, como las monedas complementarias de Venezuela y Ecuador. Después de conformado un grupo local movilizado políticamente bajo la bandera de la autonomía, éste tomó la decisión desde abajo de alivianar la radicalidad de la iniciativa de cambio, estableciendo relaciones de relativa dependencia con organismos trasnacionales que son su antípoda. No obstante, una parte de sus actuaciones siguieron comprometidas con transformaciones sustanciales como la diversificación productiva, el autoabastecimiento y la economía solidaria.

Otro problema derivado del concepto de iniciativas de transformación se refiere a los criterios para distinguir una transformación de otra que no lo es, así como para considerar cuándo ocurre distorsión. En el caso de Agrosolidaria La Celia, más que como una finalidad compacta, la transformación se muestra más como la puesta en práctica de un "Discurso para la Transición” (Escobar, 2016a), con diferentes niveles de realización y contingencia. Las narrativas de algunos de sus asociados revelan críticas al statu quo acompañadas del reclamo de un cambio cultural a largo plazo que tome en cuenta la especificidad y los ritmos del lugar.

Paradójicamente, sus formas emergentes de materializar este pensamiento crítico tienden a reforzar -en ocasiones- el statu quo. O, en todo caso, 
cobran cierto nivel de "ecocinismo" (Cuesta, 2009) que nos recuerda la descripción que hace Tonkinwise (2014 citado en Escobar, 2016a: 157) de los agentes de cambio:

Ser un diseñador para la transición significa adoptar diferentes valores y perspectivas. Es, por lo tanto, un proceso de aprendizaje y, por la misma razón, un desafío. Requiere que los diseñadores reconozcan la hipocresía que surge de ser un agente de cambio hacia un nuevo sistema desde dentro del sistema antiguo.

Según la evidencia construida, estos procesos de aprendizaje suelen ocurrir, de forma acelerada, ligados a situaciones de crisis o emergencia, que catalizan la llegada de nuevos agentes, propuestas y discursos, con la pretensión de detonar ideas de cambio. La ausencia de gradualidad en el diseño de las iniciativas representa una especie de trampa para la acción colectiva, pues -como se observó en el caso de Agrosolidaria- puede terminar cayendo en el juego de agentes externos que tienen experiencia en capturar las iniciativas. Vemos entonces como, a nivel local, altas expectativas de transformación tienden a empobrecerse cuando no alcanzan a dotarse de estrategias, reglas, subsidios y demás mecanismos que permitan vigilar las interacciones con la modernización. No deja de resultar paradójico que el proceso de acompañamiento ofrecido por la Fundación Newman operara soportado en la fortaleza tramas comunitarias, pero perdiera eficacia justo cuando estas se fragmentaran.

El estudio de caso presentado permite concluir que la debilidad del asociacionismo parece no residir en el familiarismo -como sostienen Valenzuela y Causiño (2000)-, sino más bien en abruptos procesos de adaptación que experimentan las asociaciones en su interacción con propuestas desarrollistas. Exponerse a esta interacción tiende a dejar instalada una atmósfera de sospecha, de amenaza, de recelo, de promesas incumplidas y de trampas, que termina siendo absorbida por las asociaciones y las sociabilidades que le son constitutivas. Finalmente es en la esfera privada, familiar y comunitaria, donde los pequeños agricultores asociados deben recomponer decepciones y vínculos sociales fragmentados, además de continuar sobreviviendo autónomamente en tiempos de mercados pobres. Se trata de fracasos temporales, no superiores a la necesidad humana de confiar en otras personas, que sin embargo nos muestran algunas claves para comprender el tan manido dicho de que "a la gente le cuesta asociarse". 


\section{RECONOCIMIENTOS}

Este artículo es resultado de la investigación postdoctoral "Iniciativas de Transformación y Asociatividad en comunidades rurales andinas", financiado por Ministerio de Ciencia, Tecnología e Innovación de Colombia y desarrollada en la empresa Innogest S.A.S. Forma parte de un proyecto de investigación más amplio en colaboración con un equipo de la Universidad de Varsovia, dirigido por Agata Hummel. La autora agradece a los financiadores de la estancia postdoctoral, así como a la empresa Innogest S.A.S y al equipo científico polaco que la acompaña. El artículo hace uso de materiales derivados de la tesis doctoral "Hacer espacios en patrimonialización. Prácticas cotidianas en la formación del 'Paisaje Cultural Cafetero' de Colombia - Patrimonio Mundial", elaborada por la autora y sustentada en la Universidad de Valencia (España), en 2017, bajo la supervisión de Macarena Hernández Ramírez y Beatriz Santamarina Campos, a quienes la autora agradece y reconoce su contribución. También agradece el apoyo en terreno prestado por el equipo científico de Wildlife Conservation Society - Programa Colombia, la Alcaldía de La Celia, la Asociación Agrosolidaria, el Comité Municipal de Cafeteros de La Celia y la empresa Innogest S.A.S. Finalmente, agradece a los editores y evaluadores de la Revista del CESLA por sus valiosos aportes.

\section{BIBLIOGRAFÍA}

Alzate, C. A. (2019). Da desconstrução da inovação ao papel ativo da inovação social em ambientes de economia solidária na configuração de territorialidades: Proposta metodológica aplicada no estudo de caso Risaralda-Colômbia. Caminhos de Geografia, 20(70), pp. 36-55. https://doi.org/10.14393/RCG207042084

Alcaldía de La Celia. (1999). Esquema básico de ordenamiento territorial. Diagnóstico territorial. [Documento electrónico]. Manuscrito sin publicar.

Alcaldía de La Celia. (2011). Diagnóstico rural participativo. [Documento electrónico]. Manuscrito sin publicar.

Celeita, O. E. (2012). Prácticas de Comercio Justo en Colombia y programas de inclusión que permiten el desarrollo económico, social y ambiental del sector rural en el municipio de Tibasosa, Departamento de Boyacá. Estudio de caso: Agrosolidaria Confederación Colombia (trabajo de grado). Bogotá, Colombia: Universidad San Buenaventura. http://biblioteca.usbbog.edu.co:8080/Biblioteca/BDigital/68903.pdf

Comité Municipal de Cafeteros de La Celia. (2016). Estadísticas producción de cafés especiales municipio de La Celia. [Documento electrónico]. Manuscrito sin publicar.

CONPES (Consejo Nacional de Política Económica y Social, Departamento Nacional de Planeación). (2014). Política para la preservación del Paisaje Cultural Cafetero de Colombia. Documento 3803. http://paisajeculturalcafetero.org.co/static/files/Conpes1.pdf 
Cruz-Rodríguez, E. (2013). "Todos somos hijos del café": sociología política del Paro Nacional Cafetero. Entramado, 9(2), pp. 138-158.

Cardona, J. F., Hernández, C. A., y Mosquera, J. (2015). Análisis sobre desarrollo administrativo y tecnológico de asociaciones agroindustriales, de servicios y financieras en municipios del departamento de Risaralda. Colección Académica de Ciencias Estratégicas, 2(2), pp. 1-19.

Cuesta, J. A. (2009). Ecocinismos. El medio ambiente natural y humano bajo la linterna cínica (tesis doctoral). Salamanca, España: Universidad de Salamanca.

DANE (Departamento Administrativo Nacional de Estadística). (2005). Resultados del censo general 2005. http://www.dane.gov.co/index.php/poblacion-y-demografia/proyecciones-de-poblacion

Dicum, G. (2003). Colony in a cup. Gastronomica: The Journal of Food and Culture, 3(2), pp. 71-77. https://doi.org/10.1525/gfc.2003.3.2.71

DNP (Departamento Nacional de Planeación). (2005). Índice de pobreza multidimensional por municipio y departamento, con base en el censo general de 2005. https://n9.cl/hok8

DNP (Departamento Nacional de Planeación). (2014). Política para la preservación del Paisaje Cultural Cafetero de Colombia. Documento 3803. http://paisajeculturalcafetero.org.co/static/files/Conpes1.pdf

DNP (Departamento Nacional de Planeación). (2016). Ficha Municipal de La Celia. http://ddtspr.dnp.gov.co/FIT/\#/fichas

Escobar, A. (2012). Cultura y diferencia: La ontología política del campo de cultura y desarrollo. Wale'Keru. Revista de Investigación en Cultura y Desarrollo, (2), pp. 7-16.

Escobar, A. (2016a). Autonomía y diseño. La realización de lo comunal. Popayán, Colombia: Editorial Universidad del Cauca.

Escobar, A. (2016b). Los saberes y los mundos de los pueblos-Territorio: el post-conflicto como transición civilizatoria. Conferencia - Encuentro Ecovida. Manizales, Colombia: Universidad de Caldas.

Gobernación de Risaralda. (2018). Evaluaciones agrícolas y pecuarias 2014. https://www.datos.gov.co/Agricultura-y-Desarrollo-Rural/Estadistica-2018-Municipal-Pecuario/kgru-qhfz

Gómez Cardona, S. (2010). Caficultura orgánica e identidades en el suroccidente de Colombia. El caso de la Asociación de Caficultores Orgánicos de Colombia, ACOC - Café sano (tesis de maestría). Bogotá, Colombia: Universidad Nacional de Colombia. http://www.bdigital.unal.edu.co/3141/\#sthash.4pND8MWm.dpuf

Guhl, A. (2008). Café y cambio de paisaje en Colombia, 1970-2005. Medellín, Colombia: Fondo Editorial Universidad EAFIT.

Harvey, D. (1998). La condición de la posmodernidad. Investigación sobre los orígenes del cambio cultural (trad. M. Eguía). Buenos Aires, Argentina: Amorrortu.

Hummel, A. (2019). Proyecto "Transformation Initiatives in the Andean rural communities. Narratives and strategies of social change from an anthropological perspective". Documento de trabajo. Manuscrito sin publicar.

Lombo, M. (2013). Institucionalización del género: Políticas públicas, escalas sociales y representaciones sobre mujeres cafeteras. Estudio de caso en el Colegio, Cundinamarca (tesis de maestría). Bogotá, Colombia: Universidad Nacional de Colombia. http://www.bdigital.unal.edu.co/42948/ 
London, C. (1999). Desarrollismo, democracia y crisis cafetera. Una interpretación cultural. En G. Sánchez et al. (Eds.), Conflictos regionales. La crisis del Eje Cafetero (pp. 95-149). Bogotá, Colombia: Fundación Friedrich Ebert de Colombia, FESCOL.

Ministerio de Cultura y Federación Nacional de Cafeteros de Colombia. (2012). Paisaje Cultural Cafetero. Una fusión excepcional de naturaleza, esfuerzo colectivo humano y cultura. http://paisajeculturalcafetero.org.co/static/files/dossier-pcc.pdf

Palacios, M. (1983). El café en Colombia, 1850-1970. Una historia económica, social y política. Ciudad de México, México: Ancora.

Palacios, M., y Safford, F. (2002). Colombia: país fragmentado, sociedad dividida: su historia. Bogotá, Colombia: Editorial Norma.

Rocha, R. (2014). Informalidad laboral cafetera: rasgos, determinantes y propuestas de política (Versión para comentarios, No. 011955). Bogotá, Colombia: Departamento Nacional de Planeación. https://colaboracion.dnp.gov.co/CDT/Estudios\%20Econmicos/418.pdf

Rodríguez-Herrera, D. M. (2017). Hacer espacios en patrimonialización. Prácticas cotidianas en la formación del 'Paisaje Cultural Cafetero' de Colombia - Patrimonio Mundial (tesis doctoral). Valencia, España: Universidad de Valencia.

Roseberry, W. (1996). The rise of yuppie coffees and the reimagination of class in the United $\begin{array}{llll}\text { States. American } \quad \text { Anthropologist, } & \text { 98(4), }\end{array}$ https://doi.org/10.1525/aa.1996.98.4.02a00070

Strathern, M. (Ed.). (2000). Audit cultures. Anthropological studies in accountability, ethics and the academy. Londres, Reino Unido: Routledge.

Silva, S. (2012). Evaluación de impacto de los programas de renovación de cafetales 2007-2011; efectos sobre la capacidad productiva de los caficultores colombianos. Coyuntura económica: Investigación económica y social, 42(2), pp. 15-58.

Hortons, T. (2014). Sustainability and responsability report. http://sustainabilityreport.timhortons.com/

Topik, S; Samper, M., y Talbot, J. (2012). Introducción. En S. Topik y M. Samper (Eds). Crisis y transformaciones del mundo del café: dinámicas locales y estrategias nacionales en un periodo de adversidad e incertidumbre (pp. 9-34). Bogotá, Colombia: Pontificia Universidad Javeriana.

Tulet, J.-C. (2007). Caficultores: sismogénesis de una aristocracia campesina. En B. Nates y M. Uribe (Eds.), Nuevas migraciones y movilidades (pp. 99-112). Manizales, Colombia: Centro Editorial de la Universidad de Caldas.

Valenzuela, E., y Cousiño, C. (2000). Sociabilidad y asociatividad. Un ensayo de sociología comparada. Estudios Públicos, (77), pp. 322-339.

Victoria, C. A. (2019). Las raíces históricas de la tradición asociativa de los agricultores del municipio de Apía, Risaralda, 1890-2013. Escripta. Revista de Historia, 1(1), pp. 70-94. 
